\title{
GROWTH AND YIELD RESPONSE OF MAIZE (Zea mays) TO INTER AND INTRA-ROW WeEd COMPETITION UNDER DifFerent FERTILIZER APPLICATION METHODS ${ }^{1}$
}

\author{
Crescimento e Produção do Milho (Zea mays) em Resposta à Competição com Plantas Daninhas \\ em Linha e Entrelinha sob Diferentes Métodos de Adubação
}

\author{
MAQBOOL, M.M. ${ }^{2}$, TANVEER, A. ${ }^{3}$, ALI, A. ${ }^{2}$, ABBAS, M.N. ${ }^{2}$, IMRAN, M. ${ }^{2}$, QUAYYUM, M.A. ${ }^{2}$, \\ AHMAD, M. ${ }^{4}$, and ABID, A.A. ${ }^{2}$
}

\begin{abstract}
A field experiment was conducted for two consecutive years to study the effect of fertilizer application methods and inter and intra-row weed-crop competition durations on density and biomass of different weeds and growth, grain yield and yield components of maize. The experimental treatments comprised of two fertilizer application methods (side placement and below seed placement) and inter and intra-row weed-crop competition durations each for 15, 30, 45, and 60 days after emergence, as well as through the crop growing period. Fertilizer application method didn't affect weed density, biomass, and grain yield of maize. Below seed fertilizer placement generally resulted in less mean weed dry weight and more crop leaf area index, growth rate, grain weight per cob and 1000 grain weight. Minimum number of weeds and dry weight were recorded in inter-row or intra-row weed-crop competition for 15 DAE. Number of cobs per plant, grain weight per cob, 1000 grain weight and grain yield decreased with an increase in both inter-row and intra-row weed-crop competition durations. Maximum mean grain yield of 6.35 and $6.33 \mathrm{t} \mathrm{ha}^{-1}$ were recorded in inter-row and intra-row weed competition for $15 \mathrm{DAE}$, respectively.
\end{abstract}

Keywords: competition, fertilizer, grain yield, maize, plant growth.

\begin{abstract}
RESUMO - Com o propósito de analisar que efeito os métodos de adubação e a duração da competição com planta daninha na linha e entrelinha teriam sobre a densidade e biomassa de diferentes plantas daninhas e o crescimento, rendimento de grãos e componentes na produção do milho, foi feito um estudo de campo por dois anos consecutivos. Os tratamentos experimentais incluiram dois métodos de adubação (colocação lateral e colocação por baixo das sementes) e as durações da competição com plantas daninhas na linha e entrelinha, cada qual para 15, 30, 45 e 60 dias após a emergência e durante o periodo de desenvolvimento das culturas. O método de adubação não afetou a densidade das plantas daninhas, a biomassa nem a produtividade dos grãos de milho. A colocação do adubo por baixo das sementes gerou, em grande parte, menor média de massa seca de plantas daninhas e maior índice de área com folhas na cultura, taxa de crescimento, peso de grãos por espiga e peso de mil grãos. Foram registrados o número mínimo de plantas daninhas e a massa seca em linha $e$ entrelinha com a cultura aos 15 DAE. Houve redução no número de espigas por planta, no peso de grãos por espiga, no peso de mil grãos e na produtividade de grãos com o aumento da duração da competição tanto na entrelinha como na linha. Foram registradas as produtividades médias máximas de grãos de 6,35 e 6,33 t ha l $^{-1}$ para competição com plantas daninhas em entrelinha e linha, respectivamente, aos $15 \mathrm{DAE}$.
\end{abstract}

Palavras-chave: competição, adubação, produtividade de grãos, milho, desenvolvimento das plantas

Recebido para publicação em 28.3.2015 e aprovado em 28.9.2015.

2 Ghazi University, Dera Ghazi Khan, Pakistan, <uafanser@gmail.com>; ${ }^{3}$ University of Agriculture, Faisalabad, Pakistan; ${ }^{4}$ Agricultural Training Institute, Karor Lal Eason, Layya, Pakistan. 


\section{INTRODUCTION}

Although Maize (Zea mays) is a vigorous and tall growing plant, it is susceptible to competition from weeds, with commonly reported yield losses greater than 30\% (Chikoy \& Ekeleme, 2003; Hassan et al., 2010). Enhanced crop competitiveness can be achieved either by specific breeding programme (Knezevic et al., 2002) or through changing crop husbandry, such as adjusting the time of planting (Rasheed et al., 2004), inter and intra-row hoeing (Abouziena et al., 2008), growing of crop cultivars with vigorous growth (Akmal et al., 2010) and fertilizer rate and placement (Bibi et al., 2010). The effect of all these factors depends greatly on crop species, type and level of weed infestation and environmental conditions (Hussein et al., 2007). Identifying factors that could affect crop competitive ability independently or synergistically with known factors over a wide range of situations is therefore important to enhance crop competitive ability (Tomar et al., 2003).

Maize is very sensitive to weed competition from early stages of growth (Kumar \& Sundari, 2002). It is generally assumed that weeds present within maize rows are harmful for its growth and development, since weeds compete with crop for nutrients, water and space (Movaghatiana \& Khorsandib, 2014). It has also been demonstrated that at early stages of cropweed competition, weeds located nearer to or in between crop rows are most critical (Ullah et al., 2008). Competition between crops and weeds for nutrients depends largely on total amount and types of nutrient present and their timely availability. Management practices, such as fertilizer placement that can alter nutrient availability, can greatly affect weed infestation and crop competitive ability (Rajcan \& Swanton, 2001). However, very little is known about the effect of fertilizer placement with particular reference to time of weed removal in maize. The present study has thus been conducted to determine the effect of inter and intra-row weed-crop competition durations on growth and yield of maize under different fertilizer application methods.

\section{MATERIALS AND METHODS}

This present study was conducted for two consecutive years. Five inter and intra-row weed-crop competition durations each for 15 , 30, 4560 days after emergence (DAE) and for full season under two fertilizer placement methods, namely, side placement and below seed placement, tested using the split-plot design with four replications. Fertilizer application methods were randomized in main plots, while inter-row and intra-row competition durations were randomized in sub-plots. 'Dahklab 919' was planted manually in $75 \mathrm{~cm}$ spaced rows at plant-to-plant distance of $20 \mathrm{~cm}$. Two seeds per hill were used to ensure the desired crop stand in each treatment and when plants attained four fully expanded leaves, thinning was conducted to adjust plant population. Each sub-plot was $7 \mathrm{~m}$ long and consisted of four crop rows. Crop was fertilized at the rate of $150 \mathrm{~kg} \mathrm{~N}, 100 \mathrm{~kg} \mathrm{P}$ and $100 \mathrm{~kg} \mathrm{k} \mathrm{ha}^{-1}$ applied in the form of urea, diammonium phosphate (DAP) and potassium sulphate (SOP), respectively. Half $\mathrm{N}$ and whole $\mathrm{P}$ and $\mathrm{K}$ were applied at the time of planting. In side placement treatment the fertilizer was side dressed at a distance of $5 \mathrm{~cm}$ from crop row; while in below seed placement, first fertilizer was drilled and covered with $5 \mathrm{~cm}$ soil and then seed was planted in the same row manually. Remaining half dose of $\mathrm{N}$ was side dressed in both methods at a distance of $5 \mathrm{~cm}$ from plant rows before $2^{\text {nd }}$ irrigation (8 DAS).

In inter-row weed-crop competition weeds present at $7.5 \mathrm{~cm}$ distance from each side of row were allowed to compete with crop for a specified period. Weeds from remaining areas were removed manually 30 DAS. In intra-row weed-crop competition weeds present at 7.5 distances from each side of row were removed manually and those present in remaining spaces were allowed to compete with maize. After specified weed competition duration, number of weeds was recorded from a randomly selected area measuring $0.15 \mathrm{~m} \times 6.75 \mathrm{~m}$ from each plot. Weeds from the same area were then harvested at ground level, dried at $80{ }^{\circ} \mathrm{C}$ to determine constant dry weight. High natural weed populations were observed during both years. Dominant weeds included horse purslane weed (Trianthema portulacatrum), 
purple nut sedge (Cyperus rotundus), rice (Echinochloa Colona).

Five randomly selected plants from each plot were used to determine crop growth rate and leaf area. Four measurements starting at 30 DAE with an interval of 15 days were made. Portable leaf area meter (Licor Model 3100, Nebraska, USA) was used to measure leaf area. Leaf area index (LAI) was calculated using the formula given by Beadle (1987).

Leaf area index = Leaf area/Land area

Crop Growth Rate (CGR) was calculated as increase in crop dry weight in $\mathrm{g} \mathrm{m}^{-2}$ day $^{-1}$ (Beadle, 1987).

$$
C G R=W 2-W 1 / T 2-T 1
$$

Where $W 1=$ Total dry weight at first harvest; $W 2=$ Total dry weight at second harvest; $T 1=$ Time corresponding to first harvest (days); T2 $=$ Time corresponding to second harvest (days).

At maturity, three crop rows from each plot were used to record yield and yield components. Total number of plants and cobs in these three rows were counted before harvesting. Plants were harvest at ground level and sun-dried for a week then cobs were removed from plants, shelled manually after dehusking and drying and the grain weight was recorded. A sub-sample of 1000 grains was taken, and weighed. Number of grains were calculated and multiplied by number of rows. Weight per cob was obtained by dividing total grain weight by the number of cobs harvested in each sub-plot. Statistical package MSTAT $C$ was used for statistical analysis $(p>0.05)$ and Least Significant Difference (LSD) test was used to separate the significant means (Steel $\&$ Torri, 1984).

\section{RESULTS AND DISCUSSION}

\section{Total weed density $\left(\mathrm{m}^{-2}\right)$}

Data regarding weed density per unit area presented on Table 1 show that effect of fertilizer application methods on weed density was non-significant. However, duration of weed competition had significant effect on total weed density per unit area. Interactive effects of fertilizer application methods and weed competition duration in respect to total weed density was also significant during both years of study. On the $1^{\text {st }}$ year, maximum number of weeds (106.00 plants $\mathrm{m}^{-2}$ ) was recorded in plots where inter and intra-row weeds competed with crop for full season under side placement of fertilizer $\left(\mathrm{P}_{1} \mathrm{C}_{11}\right)$ and was statistically at par with weedy check with below seed placement of fertilizer $(10 \%)$ and intra-row weed competition until maturity of crop with below seed placement of fertilizer (33\%). Weed density per unit area was statistically minimum when either inter-row or intra-row weeds competed with crop for 15 DAE in combination with any fertilizer application methods $\left(\mathrm{P}_{2} \mathrm{C}_{1}, \mathrm{P}_{2} \mathrm{C}_{6}, \mathrm{P}_{1} \mathrm{C}_{1}\right.$ and $\left.\mathrm{P}_{1} \mathrm{C}_{6}\right)$. A quite similar trend was noted on the $2^{\text {nd }}$ year.

\section{Dry weight of weeds $\left(\mathrm{g} \mathrm{m}^{-2}\right)$}

The dry matter accumulation reflects the growth behavior of weeds and gives better indication of weed-crop competition. Greater weed dry weight also reflects more utilization of soil and environmental resources by weeds at the expense of crop growth. Data presented on Table 1 show that weed dry weight was significantly affected by different inter and intra-row weed competition duration, while fertilizer application methods did not show any statistical effect on this parameter throughout both years.

The interactive effects of both these factors were also significant in the $1^{\text {st }}$ year but non-significant in the $2^{\text {nd }}$ year. Mean values indicate that maximum dry weight of weeds was obtained as a result of full season inter and intra-row weed competition in combination with any of the fertilizer application methods $\left(\mathrm{P}_{1} \mathrm{C}_{11} \& \mathrm{P}_{2} \mathrm{C}_{11}\right)$ studied. Dry weight of weeds was statistically similar when either inter-row or intra-row weeds competed with maize for $15 \mathrm{DAE}$ in either of fertilizer application methods $\left(\mathrm{P}_{2} \mathrm{C}_{1}, \mathrm{P}_{2} \mathrm{C}_{6}, \mathrm{P}_{1} \mathrm{C}_{1}\right.$ and $\left.\mathrm{P}_{1} \mathrm{C}_{6}\right)$.

\section{Leaf area index}

Leaf area index (LAI) of maize recorded in $1^{\text {st }}$ year and $2^{\text {nd }}$ year presented in Table 2 show that effect of fertilizer application methods on LAI was significant during $1^{\text {st }}$ year but it was non-significant in $2^{\text {nd }}$ year. Effects of different inter and intra-row weed 
Table 1 - Effect of fertilizer application methods and inter and intra-row weed competition durations methods on weed density and dry weights

\begin{tabular}{|c|c|c|c|c|c|c|}
\hline \multirow{2}{*}{ Treatment } & \multicolumn{3}{|c|}{ Total weed density $\left(\mathrm{m}^{2}\right)$} & \multicolumn{3}{|c|}{ Dry weight of weeds $\left(\mathrm{g} \mathrm{m}^{-2}\right)$} \\
\hline & $1^{\text {st }}$ year & $2^{\text {nd }}$ year & Mean & $1^{\text {st }}$ year & $2^{\text {nd }}$ year & Mean \\
\hline \multicolumn{7}{|c|}{ A) Fertilizer application methods } \\
\hline $\mathrm{P}_{1}=$ side placement & 82.29 & 85.36 & 83.38 & 50.03 & 49.13 & $49.58 \mathrm{a}$ \\
\hline $\mathrm{P}_{2}=$ below seed placement & 80.22 & 83.02 & 81.63 & 40.58 & 45.23 & $45.41 \mathrm{~b}$ \\
\hline LSD & --- & --- & ---- & ---- & ---- & 1.04 \\
\hline \multicolumn{7}{|c|}{ B) Weed competition duration } \\
\hline \multicolumn{7}{|l|}{ Inter-row competition for } \\
\hline $\mathrm{C}_{1}=15 \mathrm{DAE}$ & $38.75 \mathrm{f}$ & $43.13 \mathrm{e}$ & $40.94 \mathrm{f}$ & $4.18 \mathrm{~h}$ & $4.26 \mathrm{f}$ & $4.22 \mathrm{~h}$ \\
\hline $\mathrm{C}_{2}=30 \mathrm{DAE}$ & $82.13 \mathrm{~d}$ & $81.00 \mathrm{c}$ & $81.56 \mathrm{~d}$ & $41.34 \mathrm{f}$ & $41.86 \mathrm{de}$ & $41.60 \mathrm{f}$ \\
\hline $\mathrm{C}_{3}=45 \mathrm{DAE}$ & $90.63 \mathrm{c}$ & $98.50 \mathrm{ab}$ & $94.56 \mathrm{c}$ & $46.31 \mathrm{ef}$ & $45.33 \mathrm{~d}$ & $45.92 \mathrm{e}$ \\
\hline $\mathrm{C}_{4}=60 \mathrm{DAE}$ & $92.75 \mathrm{bc}$ & $98.50 \mathrm{ab}$ & $95.63 \mathrm{bc}$ & $54.36 \mathrm{~d}$ & $55.01 \mathrm{c}$ & $54.68 \mathrm{~d}$ \\
\hline $\mathrm{C}_{5}=$ Full season & $94.88 \mathrm{bc}$ & $96.00 \mathrm{~b}$ & $95.44 \mathrm{bc}$ & $76.99 \mathrm{~b}$ & $77.16 \mathrm{~b}$ & $77.07 \mathrm{~b}$ \\
\hline \multicolumn{7}{|l|}{ Inter-row competition for } \\
\hline $\mathrm{C}_{6}=15 \mathrm{DAE}$ & $41.00 \mathrm{f}$ & $43.50 \mathrm{e}$ & $42.25 \mathrm{f}$ & $3.46 \mathrm{~h}$ & $3.27 \mathrm{f}$ & $3.36 \mathrm{~h}$ \\
\hline $\mathrm{C}_{7}=30 \mathrm{DAE}$ & $63.25 \mathrm{e}$ & $62.88 \mathrm{~d}$ & $63.06 \mathrm{e}$ & $34.87 \mathrm{~g}$ & $35.94 \mathrm{e}$ & $35.40 \mathrm{~g}$ \\
\hline $\mathrm{C}_{8}=45 \mathrm{DAE}$ & $95.25 \mathrm{bc}$ & $96.00 \mathrm{~b}$ & $95.63 \mathrm{bc}$ & $44.81 \mathrm{f}$ & $46.06 \mathrm{~d}$ & $45.43 \mathrm{ef}$ \\
\hline $\mathrm{C}_{9}=60 \mathrm{DAE}$ & $94.25 \mathrm{bc}$ & $100.6 \mathrm{ab}$ & $97.44 \mathrm{bc}$ & $51.81 \mathrm{de}$ & $53.62 \mathrm{c}$ & $52.71 \mathrm{~d}$ \\
\hline $\mathrm{C}_{10}=$ Full season & $97.13 \mathrm{~b}$ & $101.10 \mathrm{ab}$ & $99.13 \mathrm{~b}$ & $71.14 \mathrm{c}$ & $73.13 \mathrm{~b}$ & $72.14 \mathrm{c}$ \\
\hline $\begin{aligned} \mathrm{C}_{11}= & \text { Both inter and intra-row } \\
& \text { competition for full season }\end{aligned}$ & $103.9 \mathrm{a}$ & $104.90 \mathrm{a}$ & $104.4 \mathrm{a}$ & $89.77 \mathrm{a}$ & $90.05 \mathrm{a}$ & $89.91 \mathrm{a}$ \\
\hline SE & 2.02 & 2.48 & 1.60 & 1.93 & 2.27 & 1.49 \\
\hline \multicolumn{7}{|c|}{ C) Interaction } \\
\hline $\mathrm{P}_{1} \mathrm{C}_{1}$ & $41.00 \mathrm{~g}$ & $45.75 \mathrm{e}$ & $43.38 \mathrm{gh}$ & $4.65 \mathrm{k}$ & 4.47 & $4.56 \mathrm{n}$ \\
\hline $\mathrm{P}_{1} \mathrm{C}_{2}$ & $94.00 \mathrm{bcd}$ & $92.50 \mathrm{c}$ & $93.25 \mathrm{~d}$ & $37.73 \mathrm{ij}$ & 38.65 & 38.191 \\
\hline $\mathrm{P}_{1} \mathrm{C}_{3}$ & $88.50 \mathrm{~d}$ & $97.25 \mathrm{bc}$ & $92.88 \mathrm{~d}$ & 45.47 fghi & 43.11 & $44.29 \mathrm{ijk}$ \\
\hline $\mathrm{P}_{1} \mathrm{C}_{4}$ & $91.50 \mathrm{~cd}$ & $95.25 \mathrm{bc}$ & $93.38 \mathrm{~d}$ & $58.24 \mathrm{de}$ & 58.98 & $58.57 \mathrm{de}$ \\
\hline $\mathrm{P}_{1} \mathrm{C}_{5}$ & $94.75 \mathrm{bcd}$ & $92.25 \mathrm{c}$ & $93.50 \mathrm{~d}$ & $91.22 \mathrm{a}$ & 92.05 & $91.64 \mathrm{a}$ \\
\hline $\mathrm{P}_{1} \mathrm{C}_{6}$ & $43.25 \mathrm{~g}$ & $47.25 \mathrm{e}$ & $45.25 \mathrm{~g}$ & $4.50 \mathrm{k}$ & 4.11 & $4.30 \mathrm{n}$ \\
\hline $\mathrm{P}_{1} \mathrm{C}_{7}$ & $59.25 \mathrm{f}$ & $59.75 \mathrm{~d}$ & $59.50 \mathrm{f}$ & $37.86 \mathrm{ij}$ & 39.33 & $38.60 \mathrm{kl}$ \\
\hline $\mathrm{P}_{1} \mathrm{C}_{8}$ & $97.25 \mathrm{bc}$ & $94.25 \mathrm{c}$ & $95.75 \mathrm{bcd}$ & $49.63 \mathrm{fg}$ & 50.67 & 50.15 fghi \\
\hline $\mathrm{P}_{1} \mathrm{C}_{9}$ & $94.75 \mathrm{bcd}$ & $105.00 \mathrm{ab}$ & $99.88 \mathrm{bc}$ & $50.61 \mathrm{efg}$ & 52.55 & $51.58 \mathrm{fg}$ \\
\hline $\mathrm{P}_{1} \mathrm{C}_{10}$ & $95.00 \mathrm{bcd}$ & $101.30 \mathrm{abc}$ & $98.13 \mathrm{bcd}$ & $73.26 \mathrm{~b}$ & 78.22 & $75.74 \mathrm{~b}$ \\
\hline $\mathrm{P}_{1} \mathrm{C}_{11}$ & $106.00 \mathrm{a}$ & $108.50 \mathrm{a}$ & $107.3 \mathrm{a}$ & $87.35 \mathrm{a}$ & 88.26 & $87.81 \mathrm{a}$ \\
\hline $\mathrm{P}_{2} \mathrm{C}_{1}$ & $36.50 \mathrm{~g}$ & $40.50 \mathrm{e}$ & $38.50 \mathrm{~h}$ & $3.72 \mathrm{k}$ & 4.05 & $3.88 \mathrm{n}$ \\
\hline $\mathrm{P}_{2} \mathrm{C}_{2}$ & $70.25 \mathrm{e}$ & $69.50 \mathrm{~d}$ & $69.88 \mathrm{e}$ & 44.96 ghi & 45.07 & $45.01 \mathrm{hij}$ \\
\hline $\mathrm{P}_{2} \mathrm{C}_{3}$ & $92.75 \mathrm{~cd}$ & $99.75 \mathrm{abc}$ & $96.25 \mathrm{bcd}$ & $47.16 \mathrm{fgh}$ & 47.94 & 47.55 ghi \\
\hline $\mathrm{P}_{2} \mathrm{C}_{4}$ & $94.00 \mathrm{bcd}$ & $101.80 \mathrm{abc}$ & $97.88 \mathrm{bcd}$ & 50.48 efg & 51.13 & $50.80 \mathrm{fgh}$ \\
\hline $\mathrm{P}_{2} \mathrm{C}_{5}$ & $95.00 \mathrm{bcd}$ & $99.75 \mathrm{abc}$ & $97.38 \mathrm{bcd}$ & $62.75 \mathrm{~cd}$ & 62.26 & $62.51 \mathrm{~d}$ \\
\hline $\mathrm{P}_{2} \mathrm{C}_{6}$ & $38.75 \mathrm{~g}$ & $39.75 \mathrm{e}$ & $39.25 \mathrm{gh}$ & $2.42 \mathrm{k}$ & 2.56 & $2.42 \mathrm{n}$ \\
\hline $\mathrm{P}_{2} \mathrm{C}_{7}$ & $67.25 \mathrm{ef}$ & $66.00 \mathrm{~d}$ & $66.63 \mathrm{e}$ & $31.88 \mathrm{j}$ & 32.53 & $32.21 \mathrm{~m}$ \\
\hline $\mathrm{P}_{2} \mathrm{C}_{8}$ & $93.25 \mathrm{~cd}$ & $97.75 \mathrm{bc}$ & $95.50 \mathrm{bcd}$ & 39.98 hi & 41.44 & $40.71 \mathrm{jkl}$ \\
\hline $\mathrm{P}_{2} \mathrm{C}_{9}$ & $93.75 \mathrm{bcd}$ & $96.25 \mathrm{bc}$ & $95.00 \mathrm{~cd}$ & $53.00 \mathrm{ef}$ & 54.68 & $53.84 \mathrm{ef}$ \\
\hline $\mathrm{P}_{2} \mathrm{C}_{10}$ & $99.25 \mathrm{abc}$ & $101.00 \mathrm{abc}$ & $100.10 \mathrm{bc}$ & $69.03 \mathrm{bc}$ & 68.04 & $68.54 \mathrm{c}$ \\
\hline $\mathrm{P}_{2} \mathrm{C}_{11}$ & $101.8 \mathrm{ab}$ & $101.30 \mathrm{abc}$ & $101.50 \mathrm{ab}$ & $92.18 \mathrm{a}$ & 91.83 & $92.01 \mathrm{a}$ \\
\hline $\mathrm{SE}$ & 2.86 & 3.51 & 2.26 & 2.74 & NS & 2.11 \\
\hline
\end{tabular}


competition duration was also significant during both years of study. Maximum LAI was recorded when intra-row weeds competed with maize for $15 \mathrm{DAE}$ in combination with below seed placement of fertilizer $\left(\mathrm{P}_{2} \mathrm{C}_{6}\right)$. It was statistically at par with inter-row weed competition for $15 \mathrm{DAE}$ under below seed placement method of fertilizer $\left(\mathrm{P}_{2} \mathrm{C}_{1}\right)$. Lowest maize LAI was calculated in treatment where inter and intra-row weeds competed with maize for full growing season in combination with side placement of fertilizer $\left(\mathrm{P}_{1} \mathrm{C}_{11}\right)$.

\section{Crop growth rate}

The growth rate of maize as affected by different duration of weed competition and fertilizer application method is presented

Table 2 - Effect of fertilizer application methods and inter and intra-row weed competition duration on leaf area index and crop growth rate of maize

\begin{tabular}{|c|c|c|c|c|c|c|}
\hline \multirow{2}{*}{ Treatment } & \multicolumn{3}{|c|}{ Leaf area index } & \multicolumn{3}{|c|}{ Crop growth rate $\left(\mathrm{g} \mathrm{m}^{-2}\right.$ day $\left.^{-1}\right)$} \\
\hline & $1^{\text {st }}$ year & $2^{\text {nd }}$ year & Mean & $1^{\text {st }}$ year & $2^{\text {nd }}$ year & Mean \\
\hline \multicolumn{7}{|c|}{ A) Fertilizer application methods } \\
\hline $\mathrm{P}_{1}=$ side placement & $3.65 \mathrm{~b}$ & 4.12 & 3.88 & $23.82 \mathrm{~b}$ & $23.63 \mathrm{~b}$ & $23.73 \mathrm{~b}$ \\
\hline $\mathrm{P}_{2}=$ below seed placement & $4.12 \mathrm{a}$ & 4.16 & 4.14 & $27.67 \mathrm{a}$ & $28.82 \mathrm{a}$ & $27.75 \mathrm{a}$ \\
\hline LSD & 0.01 & - n --- & ----- & 0.04 & 0.09 & 0.05 \\
\hline \multicolumn{7}{|c|}{ B) Weed competition duration } \\
\hline Inter-row competition for & $5.23 \mathrm{a}$ & $5.32 \mathrm{a}$ & $5.28 \mathrm{a}$ & $29.74 \mathrm{~b}$ & $30.02 \mathrm{~b}$ & $29.88 \mathrm{~b}$ \\
\hline $\mathrm{C}_{1}=15 \mathrm{DAE}$ & $4.76 \mathrm{c}$ & $4.76 \mathrm{c}$ & $4.76 \mathrm{c}$ & $27.27 \mathrm{c}$ & $27.68 \mathrm{c}$ & $27.52 \mathrm{c}$ \\
\hline $\mathrm{C}_{2}=30 \mathrm{DAE}$ & $4.00 \mathrm{e}$ & $3.94 \mathrm{e}$ & $3.97 \mathrm{e}$ & $25.54 \mathrm{~d}$ & $25.83 \mathrm{~d}$ & $27.69 \mathrm{~d}$ \\
\hline $\mathrm{C}_{3}=45 \mathrm{DAE}$ & $3.51 \mathrm{~g}$ & $3.54 \mathrm{~g}$ & $3.53 \mathrm{~g}$ & $25.22 \mathrm{~d}$ & $24.33 \mathrm{f}$ & $24.78 \mathrm{f}$ \\
\hline $\mathrm{C}_{4}=60 \mathrm{DAE}$ & $3.37 \mathrm{~h}$ & $3.90 \mathrm{~h}$ & $4.63 \mathrm{~h}$ & $24.63 \mathrm{e}$ & $24.14 \mathrm{f}$ & $24.38 \mathrm{~g}$ \\
\hline $\mathrm{C}_{5}=$ Full season & $5.23 \mathrm{a}$ & $5.32 \mathrm{a}$ & $5.28 \mathrm{a}$ & $29.74 \mathrm{~b}$ & $30.02 \mathrm{~b}$ & $29.88 \mathrm{~b}$ \\
\hline \multicolumn{7}{|l|}{ Inter-row competition for } \\
\hline $\mathrm{C}_{6}=15 \mathrm{DAE}$ & $5.09 \mathrm{~b}$ & $5.12 \mathrm{~b}$ & $5.10 \mathrm{~b}$ & $30.98 \mathrm{a}$ & 30.73 a & 30.86 a \\
\hline $\mathrm{C}_{7}=30 \mathrm{DAE}$ & $4.38 \mathrm{~d}$ & $4.48 \mathrm{~d}$ & $4.43 \mathrm{~d}$ & $27.59 \mathrm{c}$ & $27.38 \mathrm{c}$ & $27.49 \mathrm{c}$ \\
\hline $\mathrm{C}_{8}=45 \mathrm{DAE}$ & $3.68 \mathrm{f}$ & $3.70 \mathrm{f}$ & $3.69 \mathrm{f}$ & $25.21 \mathrm{~d}$ & $25.82 \mathrm{e}$ & $25.25 \mathrm{e}$ \\
\hline $\mathrm{C}_{9}=60 \mathrm{DAE}$ & $3.24 \mathrm{I}$ & $3.26 \mathrm{~h}$ & $3.25 \mathrm{I}$ & $23.61 \mathrm{f}$ & $23.47 \mathrm{~g}$ & $23.54 \mathrm{~h}$ \\
\hline $\mathrm{C}_{10}=$ Full season & $3.20 \mathrm{I}$ & $3.21 \mathrm{~h}$ & $3.20 \mathrm{I}$ & $22.67 \mathrm{~g}$ & $22.86 \mathrm{~h}$ & $22.76 \mathrm{I}$ \\
\hline $\begin{aligned} \mathrm{C}_{11}= & \text { Both inter and intra-row } \\
& \text { competition for full season }\end{aligned}$ & $2.26 \mathrm{j}$ & $2.26 \mathrm{I}$ & $2.26 \mathrm{j}$ & $20.63 \mathrm{~h}$ & $21.27 \mathrm{I}$ & $20.59 \mathrm{j}$ \\
\hline $\mathrm{SE}$ & 0.03 & 0.06 & 0.03 & 0.19 & 0.15 & 0.12 \\
\hline \multicolumn{7}{|c|}{ C) Interaction } \\
\hline $\mathrm{P}_{1} \mathrm{C}_{1}$ & $0.92 \mathrm{~b}$ & $0.07 \mathrm{~b}$ & $4.97 \mathrm{~b}$ & $28.08 \mathrm{~d}$ & $27.85 \mathrm{de}$ & $27.97 \mathrm{f}$ \\
\hline $\mathrm{P}_{1} \mathrm{C}_{2}$ & $4.58 \mathrm{~d}$ & $4.56 \mathrm{c}$ & $4.57 \mathrm{c}$ & $24.94 \mathrm{~h}$ & $25.29 \mathrm{~h}$ & $25.12 \mathrm{I}$ \\
\hline $\mathrm{P}_{1} \mathrm{C}_{3}$ & $3.82 \mathrm{~g}$ & $3.73 \mathrm{f}$ & $3.78 \mathrm{~g}$ & $23.90 \mathrm{ij}$ & $23.51 \mathrm{ij}$ & $23.70 \mathrm{k}$ \\
\hline $\mathrm{P}_{1} \mathrm{C}_{4}$ & $3.15 \mathrm{jh}$ & $3.16 \mathrm{hi}$ & $3.16 \mathrm{k}$ & $23.49 \mathrm{jk}$ & $22.17 \mathrm{jk}$ & $22.83 \mathrm{~lm}$ \\
\hline $\mathrm{P}_{1} \mathrm{C}_{5}$ & $3.10 \mathrm{j}$ & $3.15 \mathrm{j}$ & 5.621 & $22.63 \mathrm{I}$ & $22.16 \mathrm{I}$ & $22.40 \mathrm{~m}$ \\
\hline $\mathrm{P}_{1} \mathrm{C}_{6}$ & $4.64 \mathrm{~cd}$ & $4.65 \mathrm{c}$ & $4.65 \mathrm{c}$ & $29.64 \mathrm{c}$ & $29.21 \mathrm{c}$ & $29.42 \mathrm{e}$ \\
\hline $\mathrm{P}_{1} \mathrm{C}_{7}$ & $3.99 \mathrm{f}$ & $4.02 \mathrm{de}$ & $4.01 \mathrm{e}$ & $24.36 \mathrm{hi}$ & $24.36 \mathrm{hi}$ & $24.36 \mathrm{j}$ \\
\hline $\mathrm{P}_{1} \mathrm{C}_{8}$ & $3.55 \mathrm{~h}$ & $3.47 \mathrm{~g}$ & $3.51 \mathrm{I}$ & $22.93 \mathrm{kl}$ & $23.07 \mathrm{kl}$ & 23.001 \\
\hline $\mathrm{P}_{1} \mathrm{C}_{9}$ & $3.07 \mathrm{j}$ & $3.08 \mathrm{ij}$ & $3.07 \mathrm{kl}$ & $21.44 \mathrm{~m}$ & $24.41 \mathrm{~m}$ & $21.43 n$ \\
\hline $\mathrm{P}_{1} \mathrm{C}_{10}$ & $3.09 \mathrm{j}$ & $3.13 \mathrm{hi}$ & $3.11 \mathrm{kl}$ & $20.23 \mathrm{n}$ & $20.52 \mathrm{n}$ & 20.37 o \\
\hline$P_{1} C_{11}$ & $2.23 \mathrm{k}$ & $2.32 \mathrm{k}$ & $2.28 \mathrm{~m}$ & $20.37 \mathrm{n}$ & $20.39 n$ & 20.38 o \\
\hline $\mathrm{P}_{2} \mathrm{C}_{1}$ & $5.58 \mathrm{a}$ & $5.63 \mathrm{a}$ & $5.60 \mathrm{~s}$ & $31.40 \mathrm{~b}$ & $32.19 \mathrm{~b}$ & $31.79 \mathrm{~b}$ \\
\hline $\mathrm{P}_{2} \mathrm{C}_{2}$ & $4.95 \mathrm{~b}$ & $4.98 \mathrm{~b}$ & $4.95 \mathrm{~b}$ & $29.74 \mathrm{c}$ & $30.07 \mathrm{c}$ & $29.93 \mathrm{~d}$ \\
\hline $\mathrm{P}_{2} \mathrm{C}_{3}$ & $4.18 \mathrm{e}$ & $4.15 \mathrm{~d}$ & $4.17 \mathrm{~d}$ & $27.18 \mathrm{ef}$ & $28.17 \mathrm{ef}$ & $27.67 \mathrm{f}$ \\
\hline $\mathrm{P}_{2} \mathrm{C}_{4}$ & $3.87 \mathrm{fg}$ & $3.92 \mathrm{e}$ & $3.90 \mathrm{ef}$ & $26.95 \mathrm{ef}$ & $26.50 \mathrm{ef}$ & $26.73 \mathrm{~g}$ \\
\hline
\end{tabular}

Values followed by the same letters do not differ significantly at $5 \%$ probability level. DAE $=$ Days After Emergence. 
in Table 2. The data clearly show that effect of fertilizer application methods and weed competition durations was significant on crop growth rate (CGR) during both years.

The interactive effects of both the factors studied were also significant during both years of study. Maximum CGR was recorded in intra-row weed-crop competition for $15 \mathrm{DAE}$ with below seed fertilizer placement method $\left(\mathrm{P}_{2} \mathrm{C}_{6}\right)$ in both years. In both years minimum CGR was observed in treatment where inter and intra-row weeds competed with maize throughout the season with any fertilizer application method $\left(\mathrm{P}_{1} \mathrm{C}_{11} \& \mathrm{P}_{2} \mathrm{C}_{11}\right)$ studied.

\section{Number of cobs per plant}

It is evident from data (Table 3) that effect of fertilizer application methods on number of cobs per plant was non-significant in $1^{\text {st }}$ year and significant in $2^{\text {nd }}$ year. Mean values indicate that significantly maximum number of cobs per plant was recorded in below seed fertilizer placement method $\left(\mathrm{P}_{2}\right)$ as compared with side placement method $\left(\mathrm{P}_{1}\right)$. Data also shows that duration of inter and intra-row weed competition had significant effect on this parameter during both years. In $1^{\text {st }}$ year, maximum number of cobs per plant was counted in inter-row weed competition for 15 DAE $\left(\mathrm{C}_{1}\right)$. It was statistically at par with intrarow weed competition for $15 \mathrm{DAE}\left(\mathrm{C}_{6}\right)$ and interrow weed competition for $30 \mathrm{DAE}\left(\mathrm{C}_{2}\right)$. Significantly lesser number of cobs per plant was recorded in weedy check treatment where inter and intra-row weeds competed with maize for full season $\left(C_{11}\right)$. In $2^{\text {nd }}$ year, statistically similar number of cobs per plant was recorded when either inter-row or intrarow weeds competed with maize for $15 \mathrm{DAE}$.

The interactive effect of fertilizer application methods and weed competition duration was found to be non-significant during both years, however two-year average data show significant effect. Maximum number of cobs per plant was recorded in plots where inter-row weeds competed with maize for $15 \mathrm{DAE}$ in combination with side placement of fertilizer $\left(\mathrm{P}_{1} \mathrm{C}_{1}\right)$.

Minimum number of cobs per plant was recorded in plots where inter and intra-row weeds competed with maize for full season in combination with any fertilizer application method $\left(\mathrm{P}_{1} \mathrm{C}_{11}\right.$ \& $\left.\mathrm{P} 2 \mathrm{C} 11\right)$.

\section{Grain weight per cob (g)}

Grain weight per cob was significantly affected by fertilizer application methods and duration of inter and intra-row weed competition (Table 3 ). Their interactive effects were also significant during both year of study. In $1^{\text {st }}$ year, maximum grain weight per cob (93.47 g) was recorded in below seed fertilizer placement method in combination with intra-row weed competition for $15 \mathrm{DAE}$ $\left(\mathrm{P}_{2} \mathrm{C}_{6}\right)$. It was statistically at par with inter-row weed competition for $15 \mathrm{DAE}$ with below seed placement of fertilizer $\left(\mathrm{P}_{2} \mathrm{C}_{1}\right)$. These were followed by below seed placement of fertilizer in combination with either inter-row or intrarow weed competition for $30 \mathrm{DAE}\left(\mathrm{P}_{2} \mathrm{C}_{2} \& \mathrm{P}_{2} \mathrm{C}_{7}\right)$. The significantly minimum grain weight per cob was noted in full season competition of inter and intra-row weeds under side placement of fertilizer $\left(\mathrm{P}_{1} \mathrm{C}_{11}\right)$. Almost similar trend was noted in $2^{\text {nd }}$ year, except statistically similar values for grain weight per cob in inter-row weed competition for 15 and $30 \mathrm{DAE}$ under below seed placement of fertilizer $\left(\mathrm{P}_{2} \mathrm{C}_{1}\right.$ $\& \mathrm{P}_{2} \mathrm{C}_{2}$ ).

\section{0-grain weight (g)}

The development of grains reflects the photosynthetic potential of a plant and its capacity to transport assimilates to economically valuable plant organs (Ogbaji, 2003). The data pertaining to 1000 grain weight presented in table 3reveals that fertilizer application methods and duration of inter and intra-row weed competition had significant effect on 1000 grain weight of maize. Their interactive effects were also significant in both years. In the $1^{\text {st }}$ year, heaviest grain weight was recorded in below seed fertilizer placement method in combination with either inter-row or intra-row weed competition duration for $15 \mathrm{DAE}\left(\mathrm{P}_{2} \mathrm{C}_{6} \& \mathrm{P}_{2} \mathrm{C}_{1}\right)$. The values for theses combinations were 269.90 and $268.10 \mathrm{~g}$, respectively, which were statistically at par with each other. These were followed by inter-row weed competition for $30 \mathrm{DAE}$ with 


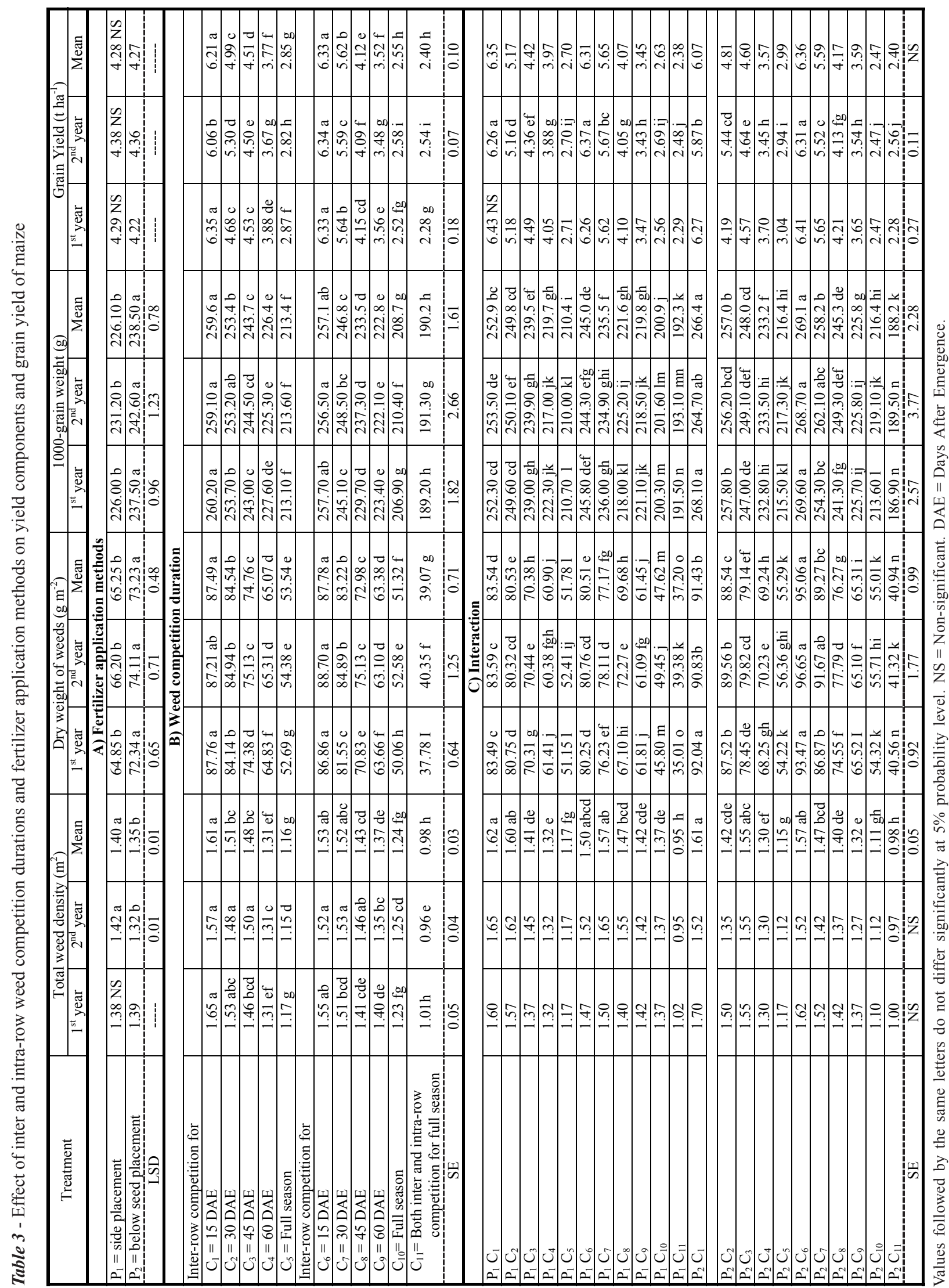

Planta Daninha, Viçosa-MG, v. 34, n. 1, p. 47-56, 2016 
below seed fertilizer placement method $\left(\mathrm{P}_{2} \mathrm{C}_{2}\right)$. The lowest 1000-grain weight was recorded in both fertilizer application methods when weeds (inter \& intra row) competed with maize for full growing season $\left(\mathrm{P}_{2} \mathrm{C}_{11} \& \mathrm{P}_{1} \mathrm{C}_{11}\right)$. They were statistical similar to each other. Similar observations were noted in $2^{\text {nd }}$ year.

\section{Grain yield (t ha-1)}

The final grain yield is the function of combined effect of all the components under influence of a particular set of environmental conditions. Data regarding grain yield (Table 3 ) of maize as affected by fertilizer application methods was not significant during both years, however duration of inter and intra-row weed competition had significant effect on grain yield of maize during both years of study. In both years, higher grain yield $(6.35$ and $6.06 \mathrm{t} \mathrm{ha}^{-1}$, respectively in $1^{\text {st }}$ year and in $2^{\text {nd }}$ year) was recorded in treatment where intra-row weeds competed with maize for $15 \mathrm{DAE}\left(\mathrm{C}_{6}\right)$. It was statistically at par with inter-row weed-crop competition for $15 \mathrm{DAE}$ in $1^{\text {st }}$ year, but followed by the same treatment in $2^{\text {nd }}$ year. Minimum grain yield was recorded where inter and intra-row weeds competed with maize for full season $\left(C_{11}\right)$ in both years.

Interactive effect of fertilizer application methods and weed competition duration was non-significant in $1^{\text {st }}$ year but it was significant in $2^{\text {nd }}$ year. Mean values indicate that intra-row weed-crop competition for 15 DAE with side placement method $\left(\mathrm{P}_{1} \mathrm{C}_{6}\right)$ produced the maximum grain yield $\left(6.37 \mathrm{t} \mathrm{ha}^{-1}\right)$. It was statistically at par with intra-row weed-crop competition for $15 \mathrm{DAE}$ with below seed fertilizer placement method $\left(\mathrm{P}_{2} \mathrm{C}_{6}\right)$ and inter-row weed competition for 15 DAE with side placement method $\left(\mathrm{P}_{1} \mathrm{C}_{1}\right)$. The lowest $(38.77 \%)$ grain yield was recorded when intra-row weeds competed with maize for full season in combination with below seed placement of fertilizer $\left(\mathrm{P}_{2} \mathrm{C}_{10}\right)$. However, it was statistically at par with when both inter \& intra-row weeds competed with maize for full season with either of fertilizer application method $\left(\mathrm{P}_{1} \mathrm{C}_{11} \& \mathrm{P}_{2} \mathrm{C}_{11}\right)$.

Increase in weed density up till maturity of crop clearly shows that weed seeds germinated throughout the growth period of maize. Similar results were also reported by Khan et al. (2012), who concluded that weed density per unit area increases with increased crop growth. There was a linear increase in dry weight of weeds with increase in competition duration; probably because weeds used environmental resources for a longer period of time. Similar results have also been reported by Rasheed et al. (2004) and Maqbool et al. (2006), who have claimed that significant reduction in weed dry weight (DW) in plots kept weed free up to 45 DAS, where 30 DAS are the critical period for weed competition in maize.

Maize plots showed highest LAI when intra-row weeds competed with maize for $15 \mathrm{DAE}$ and fertilizer was placed below maize seed. It could be attributed to least competition offered by intra-row weeds for 15 days after emergence. This combination showed superiority over $\mathrm{P}_{2} \mathrm{C}_{1}$ that was probably due to more competition offered by inter-row weeds for $15 \mathrm{DAE}$ with same method of fertilizer application as in aforesaid treatment. Severe reduction in LAI of maize at different stages in treatment where inter and intra-row weeds competed with maize for full season was due to severe competition for environmental resources throughout the growth period of crop. This study agrees with the results of Evans et al. (2003) who stated the reduction in corn leaf area due to weed interference usually increasing with the stages of competition. These results indicate that as competition was prolonged, the availability of essential growth elements and resources to crop became limited and hence growth rate was reduced. The data also shows that weeds present within the row (intra-row) caused more reduction in CGR than inter- row weeds being present very close to maize plants. These results are in line with Irshad, (2004) who reported that weed interference with crop has suppressive effects on CGR. Highest CGR in treatment where fertilizer was placed below maize seed might have been resulted from increased nutrient availability and nutrient uptake by the crop.

Less number of cobs per plant in plots where inter and intra-row weeds competed with maize for full season can be associated with maximum weed competition, which might had decreased nutrients and moisture 
availability to maize plants. These results differ from those of Rambakudzibga et al. (2002) who reported that maize cob count were not significantly affected by weed competition when weeds were removed as late as 8 week after crop emergence. Decreasing trend in number of cobs per plant with increased inter or intra-row weed competition duration was due to increased competition between crop and weeds. The maximum grain weight per cob in intra-row weed competition for $15 \mathrm{DAE}$ with placement of fertilizer below seed was most probably due to increased LAI and CGR of maize with least intra-row weed competition. The other reason might be the absence of interrow weeds. Similar results were also reported by Saeed et al. (2010) and Shinggu et al. (2009). They reported that early weed removal can increase grain weight per cob compared with weedy check. Heaviest grain weight produced as a result of intra-row competition for $15 \mathrm{DAE}$ with placement of fertilizer below seed appeared to be quite logical as the crop fully utilized the applied fertilizer with competitive effects of intra-row weeds for a least period. Similar was the case of treatment combination $\mathrm{P}_{2} \mathrm{C}_{1}$. Stress at grain filling stage may influence the grain development system; hence due to severe stress created by weed competition lighter grains were produced in full season competition. Reduction in 1000grain weight due to weed competition was also reported by Evans et al. (2003); Talebbeigi \& Ghadiri, (2012).

Greater grain yield obtained with least intra-row or inter-row weeds competition might be due to lower weed competition duration which helped improving LAI, CGR, number of grains per plant, 1000 grain weight, grain weight per cob and consequently greater yield of maize. Similar results have also been published by Iremiren et al. (1999) and Kumar $\&$ Sundari (2002) who reported that weed free crop up to harvest shows the highest grain yield statistically at par with weedy up to 15 DAE.

\section{LITERATURE CITED}

ABOUZIENA, H. F. et al. Effect of plant spacing and weed control treatments on maize yield and associated weeds in sandy soils. Am. Eur. Agric. Environ. Sci., v. 4, n. 1, p. 9-17, 2008 .
AKMAL, M. et al. Response of maize varieties to nitrogen application for leaf area profile, crop growth, yield and yield components. Pakistan J. Bot., v. 42, n. 3, p. 1941-1947, 2010 .

MOVAGHATIANA, A.; KHORSANDIB, F. Allelopathic effects of extracts from two growth stages of wheat on wild mustard germination characteristics. Inter.J. Plant An.

Environ. Sci., v. 4, n. 3, p. 454-458, 2014.

BEADLE, C. L. Plant Growth Analysis. In: COOMLOS, J. D. O.; LONG, S. P.; SCURLOCK, J. M. O. (Eds.).

Techniques in Bio-productivity and Photosynthesis. 2.ed., p. 21-23, 1987. (Pergamon press, Oxford, NewYork)

BIBI, Z. et al. Integrating cultivars with reduced herbicides rates for weed management in maize. Pakistan J. Bot., v. 42, n. 3, p. 1923-1929, 2010.

CHIKOYE, D.; EKELEME, F. Cover crops for cogongrass management and effects on subsequent corn yield. Weed Sci., v. 51, n. 5 , p. $792-797,2003$.

EVANS, S. P. et al. Nitrogen application influences the critical period for weed control in corn. Weed Sci., v. 51, n. 3, p. 408-417, 2003.

HASSAN, G. et al. Integrating cultivars with reduced herbicides rates for weed management in maize. Pakistan J. Bot., v. 42, n. 3, p. 1923-1929, 2010.

HUSSEIN, F. et al. Effect of nitrogen rates and weed control treatments on maize yield and associated weeds in sandy soils. Weed Technol., v. 21, n. 4, p. 1049-1053, 2007.

IREMIREN, G. O. et al. Effect of spacing on the growth, yield and quality of okra in southern Nig. J. Agric. Sci., v. 10, n. 1, p. 12-26. 1999.

IRSHAD, A. et al. Effect of sorghum extract on management of barnyardgrass in rice crop. Allelopathy J., v. 14, n. 1, p. 205-212, 2004.

KHAN, N. W. et al. Integration of nitrogen fertilizer and herbicides for efficient weed management in maize crop. Sarhad J. Agric., v. 28, n. 3, p. 457-463, 2012.

KUMAR, S. M. S. et al. Studies on the effect of major nutrients and crop weed competition period in maize. Indian J. Weed Sci., v. 34, n. (3-4), p. 309-310, 2002.

KNEZEVIC, S. Z. et al. Critical period for weed control: the concept and data analyses. Weed Sci., v. 50, n. 6, p. 773-786, 2002.

MAQBOOL, M. M. et al. Growth and yield of maize (Zea mays L.) as affected by row spacing and weed competition durations. Pakistan J. Bot., v. 38, n. 4, p. 1227-1236, 2006. 
OGBAJI, M. I. Effects of N rates and intra row spacing on local maize (Zea mays L.) in the southern Guinea. J. Sustain. Agric. Environ., v. 5, n. 1, p. 147-152, 2003.

RAJCAN, I.; SWANTON, C. J. Understanding maize-weed competition: resource competition, light quality and the whole plant. Field Crops Res., v. 71, n. 2, p. 139-150, 2001.

RAMBAKUDZIBGA, A M., et al. Host -parasite relations between cowpea and Alectra vogelii. European Weed Res. Soc. Weed Res., v. 42, n. 3, 249-256. 2002.

RASHEED, M.; KHALIL, J.; HUSSAIN, M. Biological response of maize to phosphorus and planting geometry. Inter. J. Agric. Biol., v. 6, n. 3, p. 462-464, 2004.

SAEED, M. et al. Effect of nitrogen levels and weed-crop competition durations on yield and yield components of maize. J. Agric. Res., v. 48, n. 4, p. 471-481, 2010.
SHINGGU, C. P. et al. Effects of variety, crop arrangement and period of weed interference on the performance of maize grown in mixture in northern guinea savannah of nigeria.

ARPN J. Agric. Biol. Sci., v. 4, n. 2, p. 47-55, 2009.

STEEL, R. G. D.; TORRI, J. H. Principles and procedures of statistics. 2.ed. Singapore: Mc Graw Hill Book, 1984. p. $172-177$.

TALEBBEIGI, R. M.; GHADIRI, H. Effects of Cowpea living mulch on weed control and maize yield. J. Biol. Environ. Sci., v. 6, n. 17, p. 189-193, 2012.

TOMAR, R. K. et al. Effect of weed management practices on weed growth and yield of wheat in rice based cropping system under varying levels of tillaye. Ann. Plant Protec. Sci., v. 11, n. 1, p. 123-128, 2003.

ULLAH, W. et al. Impact of integratedweed management on weed and yield of maize. Pakistan J. Weed Sci. Res., v. 14, n. 3/4, p. 141-151, 2008. 